\title{
Fatigue in chronic inflammation - a link to pain pathways
}

Karine Louati ${ }^{1,2}$ and Francis Berenbaum ${ }^{1,2^{*}}$

\begin{abstract}
Fatigue is a frequent symptom in several inflammatory diseases, particularly in rheumatic diseases. Elements of disease activity and cognitive and behavior aspects have been reported as causes of fatigue in patients with rheumatoid arthritis. Fatigue could be associated with activity of inflammatory rheumatism. Indeed, biologic agents targeting inflammatory cytokines are effective in fatigue. Fatigue is also associated with pain and depressive symptoms. Different pathways could be involved in fatigue and interact: the immune system with increased levels of pro-inflammatory cytokines (interleukin- 1 and -6 and tumor necrosis factor alpha), dysregulation of the hypothalamic-pituitary-adrenal axis and neurological phenomena involving the central and autonomic nervous systems. A pro-inflammatory process could be involved in pain and behavioral symptoms. Inflammation could be a common link between fatigue, pain, and depression.
\end{abstract}

\section{Introduction}

Fatigue is usually defined as a state of exhaustion and decreased strength accompanied by a feeling of weariness, sleepiness, and irritability, with a cognitive component [1]. A physiological fatigue state, occurring after strong physical effort, sends a signal to the body to bring it to rest to rescue the exhausted tissues (that is, the muscles). Unlike normal fatigue, pathological fatigue does not improve with rest. This kind of fatigue is seen in most acute and chronic inflammatory diseases, including arthritis.

This review discusses the place of fatigue in various inflammatory diseases but also the possible link with inflammation, pain and depression. We explain this

\footnotetext{
* Correspondence: francis.berenbaum@sat.aphp.fr

'Department of Rheumatology, Assistance Publique-Hôpitaux de Paris (AP-HP), Saint-Antoine Hospital, F-75012 Paris, France

${ }^{2}$ Inflammation-Immunopathology-Biotherapy Department (DHU i2B), Pierre \& Marie Curie University Paris 06 - INSERM UMR_S 938, Paris, France
}

relationship in terms of physiopathologic mechanisms and discuss how inflammation could have a role in the three other domains - fatigue, stress or depression, and pain. We searched for articles in MEDLINE via PubMed with the key words 'inflammation', 'fatigue', 'pain', 'depression', 'rheumatologic diseases', 'chronic fatigue syndrome' and 'treatment'. The search was completed by a hand search of references of the most relevant studies or published reviews.

\section{Multidimensional and multicausal aspects of fatigue}

Fatigue is a multidimensional concept and has various causes. In rheumatic diseases, the association between fatigue and pain has been well established [2-4]. High fatigue is most often associated with high pain, and fatigue and pain seem to be synchronous $[2,5]$. The link between fatigue and disease activity is less clear. First, fatigue is clearly a symptom included in rheumatic diseases: in rheumatoid arthritis (RA), it is an important outcome to evaluate according to OMERACT [6], and it has been associated with the Disease Activity Score in 28 joints (DAS28) and the Clinical Disease Activity Index [7]. In spondyloarthritis (SpA), fatigue is part of the Bath Ankylosing Spondylitis Disease Activity Index (BASDAI) and appears more strongly related to the disease process than patient-related variables [8]. Furthermore, in anti-neutrophil cytoplasmic antibody-associated vasculitis, fatigue was associated with increased levels of C-reactive protein (CRP) [9]. However, a systematic review of fatigue in RA found no link between it and some characteristics of inflammatory activity, such as erythrocyte sedimentation rate or DAS28 [2]. Of note, Lee et al. [10] described a subgroup of RA patients with wellcontrolled disease but high persistent levels of fatigue (34\%).

These discrepancies could be explained by the variable definitions of fatigue or its multidimensional aspect. Indeed, several aspects can affect fatigue: illness-related characteristics (pain, inflammation, disease activity and 
joint damage), physical functioning (disability, healthrelated quality of life, sleep quality), cognitive and emotional impairment (anxiety and depression) and personal components (gender, age, social support, work and environment) (Fig. 1a) [2, 11-14]. The multidimensional nature of fatigue was well described by Hewlett et al. [13] in their conceptual model of the interaction between fatigue and three components - disease process, cognitive and behavior aspects, and personal life issues with a bidirectional path suggesting interrelationships among these components. In this model, pain was included in the disease process and could cause fatigue but could also interact with other factors such as the inflammatory process (responsible for pain, joint damage and disability), anemia and sleep disturbance $[2,9,13-15]$. Conversely, in RA, fatigue seemed associated more with the global assessment of RA or pain than inflammatory components such as erythrocyte sedimentation rate or swollen joint count, perhaps because of the effect of sleep disturbance or reduced physical activity on fatigue $[3,4,16,17]$. Among the other components of the Hewlett et al. model, cognitive and behavioral factors

\section{A}

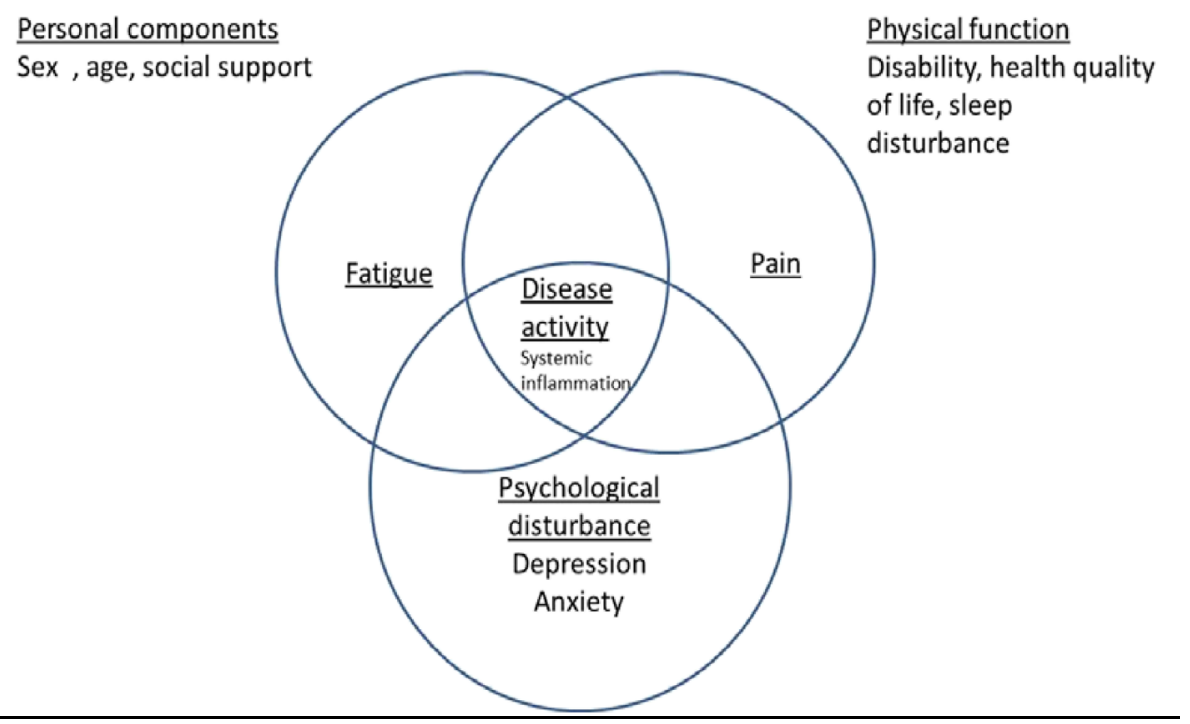

B

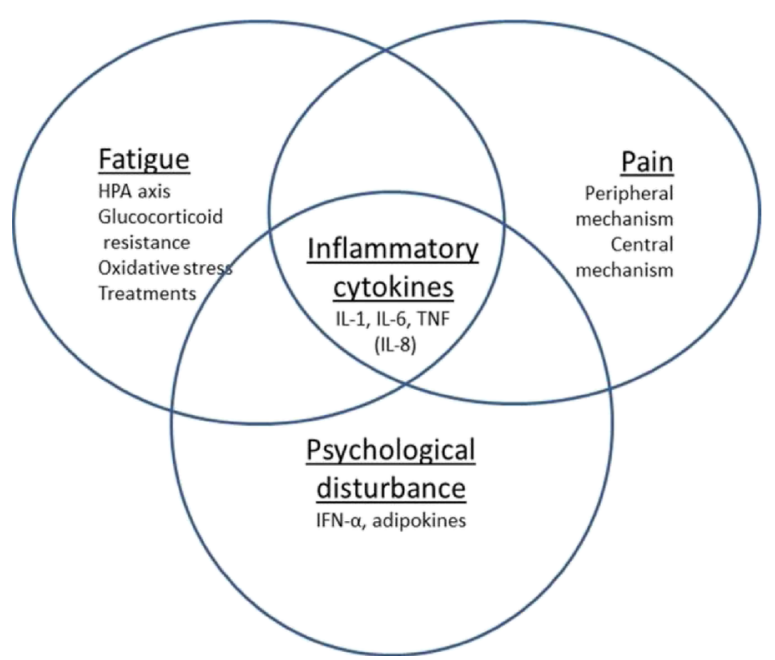

Fig. 1 Model of interactions between fatigue, pain and psychological disturbance. (a) Conceptual model of clinical interactions between fatigue, pain and psychological disturbance in inflammatory arthritis. (b) Conceptual model of physiological interactions between fatigue, pain and psychological disturbance in inflammatory rheumatism. The potential mechanisms of action in each domain are listed. The increase of inflammatory cytokine levels could be involved in fatigue, pain and mood disorders. HPA, hypothalamic-pituitary-adrenal; IFN, interferon; IL, interleukin; TNF, tumor necrosis factor 
interacted with thoughts, feelings, behaviors, and symptoms [13]. More recently, Rongen-van Dartel et al. [18] showed a high level of daily physical activity was associated with reduced fatigue even after adjustment for pain and other confounding factors. In $\mathrm{SpA}$, high fatigue was associated with the disease process, such as high disease activity (BASDAI), but also personal components, such as poor quality of life, and other disease activity (bowel symptoms) $[19,20]$. Therefore, fatigue is a subjective symptom that interacts with the multiple aspects involved in inflammatory diseases.

\section{Fatigue and inflammation}

Fatigue is common among individuals living with a chronic illness, particularly a disease with an overriding inflammatory process: rheumatologic diseases such as RA or SpA, cancers, inflammatory bowel diseases, connective tissue diseases such as systemic sclerosis, systemic autoimmune diseases, autoimmune type 1 diabetes and infections $[14,21,22]$. In a descriptive study, fatigue was more severe in patients with inflammatory bowel disease than in controls whatever their age [23].

In patients with cancer (acute myelogenous leukemia and myelodysplastic syndrome), fatigue severity was correlated with serum levels of the inflammatory cytokines interleukin (IL)-6, tumor necrosis factor (TNF)- $\alpha$ and the IL-1 receptor antagonist (IL-1RA). In lung cancer patients, IL-8 was a relevant genetic factor of pain and fatigue $[24,25]$. Moreover, the expression of IL-6 and nuclear factor-kappa B (NFkB) was increased in oncology patients with sleep disturbance [26]. In these patients, inflammation-induced fatigue could be explained by cancer treatments (radiation, chemotherapy) but also by secretion of inflammatory cytokines by the tumor [27]. Even after chemotherapy or radiation treatments, fatigue could persist for up to 10 years, when levels of a number of pro-inflammatory plasma markers, including IL-6, IL-1RA, CRP and soluble TNF receptor type II, were high $[27,28]$. A cumulative effect of levels of cytokines corresponded to the number of days with fatigue in both breast and prostate cancer patients [28]. As well, circulating $\mathrm{T}$ lymphocytes were increased in number, with no alteration in immune cells [28]. A recent review described fatigue occurring with inflammation before, during and after treatment with several cancers. Fatigue was well correlated with high levels of inflammatory peripheral cytokines (IL-6, IL-1 and TNF), which could signal the central nervous system (CNS) and generate fatigue or other behavioral symptoms [29].

For some authors, the link between inflammation and fatigue is less clear. A subgroup of patients with high fatigue and high depression harbored a minor allele for the anti-inflammatory IL-4 [30]. Moreover, fatigue was associated with some immunotherapies for HIV/AIDS, such as interferon (IFN)- $\alpha$ and IL-2; about half of 317 patients living with HIV/AIDS reported high levels of fatigue. In this study, plasma TNF levels were lower with antiretroviral therapy, CD4+ T-cell counts of at least 200 cells $/ \mathrm{mm}^{3}$ and undetectable viral loads; however, on multivariable analyses, plasma levels of none of the cytokines evaluated was significantly associated with fatigue [10].

However, the replication of studies showing an association between expression of genes or inflammation cytokines and fatigue regardless of chronic illness suggests that inflammation could play a role in the fatigue experience (Fig. 1b).

\section{Fatigue in chronic rheumatologic diseases}

Unusual and chronic fatigue with various etiologies was described for $27 \%$ of patients in a primary care clinic [31]. Fatigue is common in RA, SpA, Sjögren syndrome, systemic lupus erythematosus and vasculitis, although most publications concerned fatigue in RA or SpA [9, 14, 32-35]. In RA and SpA, the frequency of fatigue ranged from $42 \%$ to $80 \%$ depending on the definition and methods of assessment [1, 4, 8, 36-39]. For $75 \%$ of patients with ankylosing arthritis and $50 \%$ of those with RA, fatigue was considered severe [4, 39].

Several methods of evaluation have been used to investigate fatigue in rheumatologic diseases [14]. The simplest and quickest scale is the visual analog scale (VAS; scores from 0 to 100; the higher the score, the greater the fatigue), but this is a unidirectional scale. Multidirectional scales developed to include the different aspects of fatigue are the Medical Outcomes Study Short Form 36 (SF-36) vitality subscale (four questions; scores from 0 to 100; the higher the score, the lower the fatigue), the Functional Assessment of Chronic Illness Therapy Fatigue Scale (domains physical, social/family, emotional and functional; scores from 0 to 52; the higher the score, the lower the fatigue), the Profile of Mood States, the RA-specific Multidimensional Assessment of Fatigue (MAF) scale, the Multidimensional Fatigue Inventory, the Brief Fatigue Inventory, and the Fatigue Severity Scale [40-42]. With the VAS scale, the mean fatigue level in patients with RA was 42.1 [43].

Classical treatments of RA and SpA consist of pharmacological treatments to control inflammation and multidisciplinary interventions such as cognitive behavioral therapy and physical exercises to reduce symptoms and maximize self-management [44]. Among pharmacological treatments known to reduce disease activity in RA, disease-modifying antirheumatic drugs (DMARDs), such as methotrexate and leflunomide, or biologic treatments, such as anti-TNF (infliximab, adalimumab, etanercept, golimumab and certolizumab), anti-IL-6 (tocilizumab), CTLA4 immunoglobulin (abatacept) and 
anti-CD20 (rituximab), have improved pain and mood disorders as well as fatigue, although the overall effect size of biotherapies on fatigue was small (effect size $=$ 0.45 ; $95 \%$ confidence interval 0.31 to 0.58$)[4,45,46]$. More recently, new biologic therapies have shown efficacy for fatigue: FACIT-Fatigue and SF-36 vitality scales were improved with secukinumab, an antibody against IL-17, and tofacitinib, an oral Janus kinase inhibitor $[47,48]$. The placebo effect on fatigue was seen in our meta-analysis of the effect of biotherapies on fatigue: scores ranged from 1.04 $\pm 22.6 / 100$ (DEO19 study) to $11.57 \pm 21.92 / 100$ (REFLEX study) [46]. Wells et al. [49] found that 69, 84 and $90 \%$ of American College of Rheumatology 20/50/70 responders receiving abatacept, respectively, had a $>20 \%$ improvement in fatigue according to the VAS. So even if the level of concordance between fatigue and disease activity was high, $10 \%$ to a third of patients had fatigue whereas disease activity improved. With secikinumab, however, patients reporting increased pain showed worsened fatigue according to both fatigue scores [48]. After 3 months of anti-TNF therapy for RA, fatigue was decreased in patients but was independent of the level of CRP: on multiple regression, only global health and tender joint count explained $34 \%$ of the variance in fatigue [50]. Otherwise, a randomized controlled trial showed significant benefits of an exercise program on fatigue scores, quality of life, pain and sleep quality [51]. After six weekly sessions of behavioral therapy and a consolidation session, RA patients with initial VAS fatigue score $\geq 6 / 10$ reported better fatigue scores than controls (MAF and VAS scales) and better perceived fatigue severity, coping, disability, depression, helplessness, self-efficacy and sleep [52].

\section{Link between fatigue and pain}

Fatigue and pain are two common symptoms in RA, and the link between fatigue and pain in it has been described $[2-4,53]$. In a cross-sectional study, VAS fatigue score was mainly correlated with VAS pain score; pain was most strongly associated with the five variables explaining fatigue [4]. Garip et al. [53] confirmed this association, showing that fatigue intensity in RA patients was strongly correlated with VAS pain score and DAS28, with greater correlation between fatigue and pain scores than between fatigue score and DAS28. Moreover, after treatments (DMARDs or biologic agents), the decrease in VAS fatigue score was correlated with ameliorated pain and improved DAS28 score [4]. For Wolfe et al. [11], pain was a strong independent predictor of fatigue with sleep disturbance, depression, tender joint count and disability by the health assessment questionnaire. Among the variables usually linked to fatigue, pain was a better predictor of fatigue [54].

To examine the bidirectional effect of pain and fatigue, van Dartel et al. [5] conducted a prospective study of patients with established RA who received DMARDs and/or biologic agents; pain and fatigue were measured monthly for 1 year. Pain and fatigue levels fluctuated, and the change in fatigue level was positively associated with change in pain level during the same month. However, change in fatigue level was not related to a change in pain level that occurred 1 month earlier, and change in pain level was not related to a change in fatigue level 1 month earlier [5]. Moreover, in a cohort of patients with fibromyalgia, usually considered a non-inflammatory disease, pain and fatigue scores were correlated $(r=0.45$, $P<0.001)$ and, in the prospective analysis at 1 week, daily pain evaluation predicted increased fatigue level reported the next day (more than depression or daily sleep quality) [16].

\section{Fatigue and pain pathways: role of inflammation Role of inflammation in fatigue}

The mechanisms of fatigue are complex and have been studied in animal models and humans. Because fatigue could be explained by loss of muscle mass or altered mood, Norden et al. [55] proposed a model to discriminate between these phenomena: some colon tumor-bearing mice demonstrated signs of fatigue (decreased voluntary wheel-running activity) and depressed mood (resignation and anhedonia), with no association with decreased normalized contractile properties of skeletal muscle of the limb. So fatigue seemed linked more to behavior than muscle activity.

Inflammation could play an important role (Table 1). The injection of IL-1 in murine models decreased social exploration and increased hypersomnia and body weight loss, which were all improved by the administration of anti-inflammatory IL-1RA or IL-10 [56, 57]. Moreover, in the model of tumor-induced fatigue in mice seen earlier, fatigue was associated with increased levels of

Table 1 Possible mechanisms involved in fatigue

\begin{tabular}{|c|c|}
\hline \multirow[t]{2}{*}{ Endocrine system } & $\begin{array}{l}\text { Dysregulation of HPA axis and resistance to } \\
\text { glucocorticoids }\end{array}$ \\
\hline & Thyroid insufficiency \\
\hline Central nervous system & $\begin{array}{l}\text { Decrease or polymorphism of } \\
\text { neurotransmitters }\end{array}$ \\
\hline Peripheral nervous system & Alteration of autonomic system \\
\hline
\end{tabular}

Stimulation of vagus nerve via microbiota or inflammation
Anemia
Decrease of tissue oxygenation
Inflammation
Increase of levels of pro-inflammatory cytokines (IL-1, IL-6, IL-8 and TNF)
Proliferation of immune cells
Oxidative stress Excess formation of free radicals
Treatments Possible side effects

HPA hypothalamic-pituitary-adrenal, IL interleukin; TNF tumor necrosis factor 
IL-1 and IL- 6 in the brain, and treatment with minocycline, an anti-inflammatory agent, improved grip strength without reducing tumor growth or muscle mass [55].

The role of inflammation in fatigue has also been shown in patients. Indeed, in those with chronic fatigue syndrome (CFS), fatigue intensity was associated with high circulating IL-8 levels [58]. Moreover, in an observational study of military personnel with insomnia, CRP level was reduced more in the restorative sleep group than in those with persistent insomnia [59]. In RA patients, a meta-analysis of therapeutic studies showed that inhibiting levels of some pro-inflammatory cytokines by biologic agents such as anti-TNF, anti-IL-6, CTLA4 immunoglobulin or anti-CD20 significantly decreased the level of fatigue whatever the therapy [46].

Otherwise, fatigue could be due to inflammationinduced anemia by decreasing iron levels mediated by
IL-6-induced hepcidin and thyroid insufficiency or decreased hypothalamic-pituitary-adrenal (HPA) axis activity and resistance to glucocorticoids (Fig. 2 and Table 1 ) $[27,60,61]$. In this system, the release of adrenocorticotropic hormone is affected by the sleep cycle, but in some diseases, the circadian cortisol cycle is abnormally flattened [61]. Therefore, neurological phenomena could be involved in fatigue (Table 1). The role of CNS neurotransmitters was mentioned in recent reviews [27, 62, 63]: fatigue was found to be related to polymorphism in catechol-O-methyltransferase (COMT) and low levels of tryptophan, an amino acid involved in the synthesis of serotonin or impaired brain dopamine and norepinephrine transmission $[27,62,63]$. In parallel, the autonomic activity was altered in a model of fatigue induced by a cognitive task, the Kana Pick-out Test (alternating open and closed eyes): VAS fatigue score was associated with

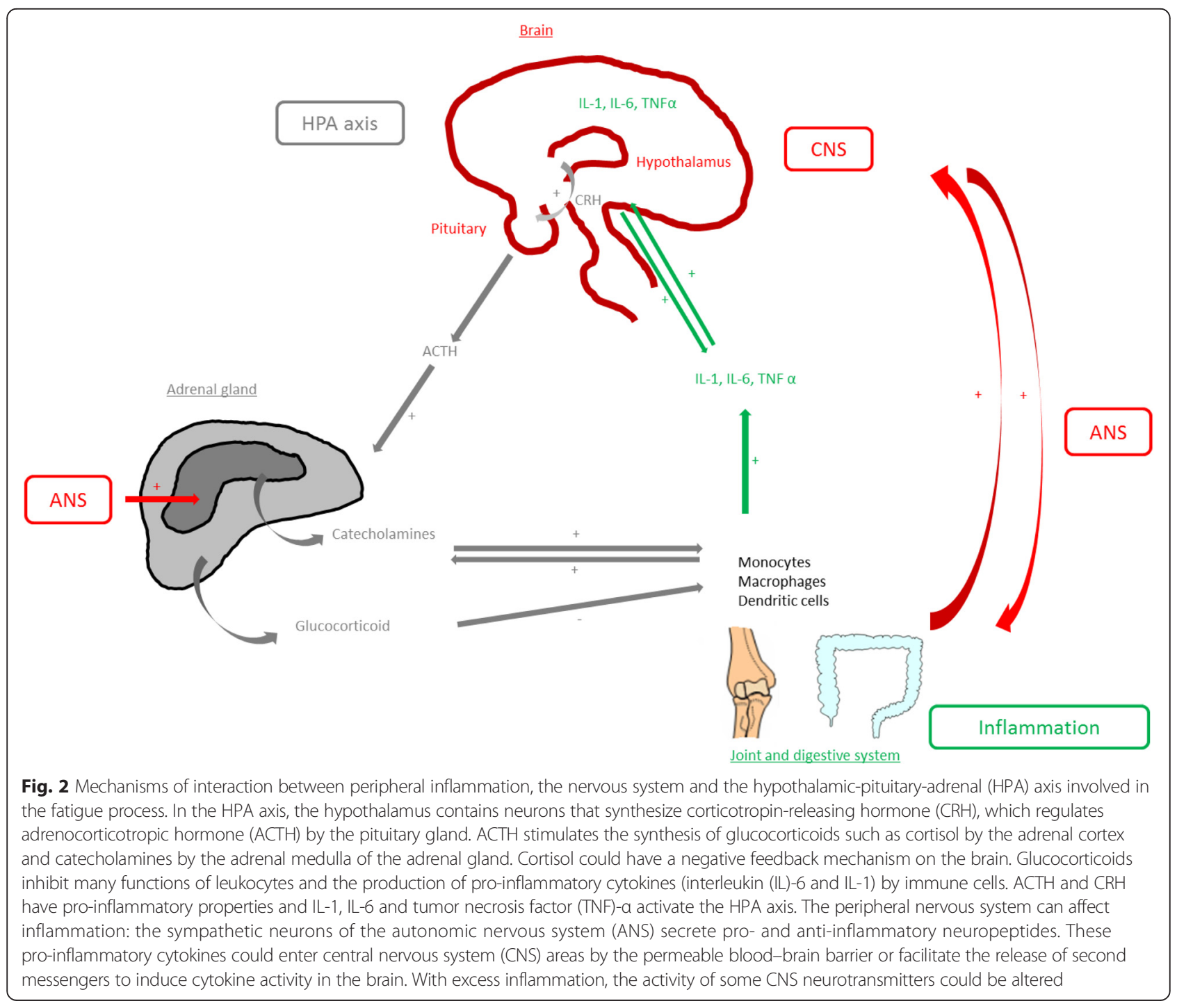


decreased parasympathetic and increased sympathetic sinus modulation as evaluated by electrocardiography [64]. Moreover, this model of induced fatigue activated the dorsolateral prefrontal cortex and cingulate cortex as assessed by functional magnetic resonance imaging (MRI) $[64,65]$.

Systemic inflammation could affect these central mechanisms. Under some circumstances, such as chronic anxiety, posttraumatic stress, and local or general inflammation diseases, the HPA axis was deregulated and the persistent secretion of corticoids induced glucocorticoid resistance [66]. The HPA axis has also been shown to interact with the immune system (Fig. 2) [61]. Moreover, although the brain is considered an immunologically privileged site, systemic infection or inflammation can have a profound effect on the CNS. In an animal model of inflammation, the peripheral administration of lipopolysaccharide increased IFN-stimulated genes in the brain [66, 67]. Peripheral proinflammatory cytokines could have a direct action when they enter CNS areas where the blood-brain barrier is permeable and an indirect action when they facilitate the release of second messengers to induce cytokine activity in the brain or when they activate the vagus or other afferent nerves [14]. TNF- $\alpha$ could participate in microglial activation in promoting rolling and adhesion of leukocytes along cerebral endothelial cells, which negatively affects dopaminergic neurotransmission [27, 63, 66-68]. However, anti-TNF agents are unable to penetrate the blood-brain barrier [69]. Inflammatory cytokines would also be responsible for a relative deficit in tetrahydrobiopterin used in the synthesis of the neurotransmitters dopamine, norepinephrine and serotonin [63]. The CNS releases norepinephrine, which is responsible for upregulating IL-1, IL-6 and TNF [66]. However, most studies examined acute inflammation, and the role of neurotransmitters in chronic inflammation is not well established. A bidirectional interaction between the neuroendocrine system and peripheral inflammation could play a role in fatigue.

\section{Role of inflammation in pain}

Pain has been investigated in animal models and humans. In animal models, pain could result from complex interactions between joint inflammation and altered pain processing: a peripheral mechanism (for example, increased innervation of the synovium; increased dorsal root ganglia expression of substrance $\mathrm{P}$, calcitonin generelated peptide and neuropeptide $Y$; increased expression of tyrosine kinase receptor A for nerve growth factor and neuronal death) and central mechanism (for example, nociceptive pathway activity, increased sensitivity of spinal neurons via glian, and activation via interleukin (IL-1, IL-6 and TNF), opiod expression in ganglia, central sensitization). Dopamine and serotonin systems are also involved in pain: COMT gene expression and polymorphisms of serotonin transporter genes were found associated with pain [62]. Patients with RA showed production of peripheral pain agents, proinflammatory cytokines (IL-1, IL-6 and TNF with different actions on responsiveness of $\mathrm{A} \delta$-fibers, $\mathrm{C}$-fibers and the effect of neutralization on mechanical hyperalgesia) and nerve growth factor in synovium or synovial fluid, which sensitized peripheral receptors [70]. TNF- $\alpha$ injected in mouse joints induced persistent sensitization of nociception with noxious stimuli, with a dosedependent effect, with prevention by injection of an antiTNF agent [71]. Endogenous opioids, somatostatin, lipid mediators and anti-inflammatory cytokines (IL-4 and IL10) were also present in synovial tissue, but their roles remain to be determined. Central pain processing was increased in RA patients, with a change in neuronal adaptive response and increased activity of the thalamus, secondary sensory cortex and limbic system, which could be modulated by emotional processing or low mood [70, 72]. Proinflammatory cytokines could have a direct action on pain via sensory neurons or an indirect action via inflammatory mediators such as prostaglandins [70].

\section{Role of inflammation in altered central nervous system activity}

Because fatigue is also often associated with anxiety and depression in inflammatory rheumatism, it may be due in part to a neurological phenomenon. Pro-inflammatory cytokines could be involved: administration of IL-1 $\beta$, IL- 6 or TNF- $\alpha$ in mice increase behavioral symptoms such as social exploration [56, 68]. A review showed that blood levels of some inflammatory cytokines, such as mitogen-stimulated cytokines and adipokines, were higher with depression [68]. A meta-analysis of 24 publications reporting on levels of cytokines in depressed patients found increased levels of TNF- $\alpha$ and IL- 6 but not IL-1 $\beta$, IL-4, IL-2, IL-8, IL-10 or IFN- $\gamma$ [73]. In some of these studies, however, this association could represent a subset of patients; for example, those with a high degree of depression, who were older and had comorbidities [74]. In older patients of the Rotterdam study, despite no association between blood levels of IL-6 and CRP and depressive symptoms, high levels of these inflammatory proteins predicted depressive symptoms 5 years later [74]. Moreover, in pooling the data for five placebo-controlled trials, Iyengar et al. [75] showed that treatment with nonsteroidal anti-inflammatory drugs (the ibuprofen or naproxen group and the celecoxib group) was associated with decreased depression score and showed a trend to changed classification of depression at 6 weeks. Moreover, antidepressive agents might inhibit the production of pro-inflammatory IL-6 and stimulate antiinflammatory IL-4, IL-10 and IL-1RA [76]. However, levels of the proinflammatory cytokines could also be altered by stressors or lifestyle factors associated with depression. 
Indeed, stress caused by major life events such as interpersonal loss or social rejection was associated with levels of pro-inflammatory IL- 6 and TNF- $\alpha$ and also CRP, especially in depressed patients [73].

Stress was also associated with high levels of the proinflammatory intracellular transcription factors $\mathrm{NF}_{\kappa} \mathrm{B}$ and inhibitor of $\mathrm{kB}$ and modulated genome-wide expression levels [66]. Thus, inflammation and depression seem to be linked, but which one affects the other is difficult to distinguish and probably there is an interaction between both.

\section{Inflammation: a potential link between fatigue, depression and pain}

Although fatigue, stress or depression, and pain have complex and various mechanisms of action, some inflammatory cytokines are found associated with these three domains, so inflammation may be their potential link. The association between these symptoms has been documented in various medical conditions: classical inflammatory diseases such as rheumatic diseases, cancer or infections but also CFS, metabolic disorders or depression, which exhibit low-grade inflammation [77].

Some authors found that in RA, fatigue, mood disorders and pain are clinically associated [2]; IL-1 level was higher in cerebrospinal fluid of patients than controls and the increased level was correlated with fatigue [78]. Biologic treatments are efficacious and can decrease levels of markers of inflammation such as CRP; tocilizumab is especially efficacious because of its action on IL-6 involved in the synthesis of CRP [79]. Thus, decreased systemic inflammation could be one of the mechanisms of action improving fatigue, pain and mood disorders.

Moreover, in a cohort of 1,466 European patients with advanced cancer, increased CRP level was associated with pain and fatigue (rho $=0.154$ and 0.197 , respectively) [80]. In ovarian cancer patients, diurnal and nocturnal cortisol and plasma IL-6 levels decreased (became normal) during the first year following surgery, and this decrease was associated with decreased fatigue and depression [81].

Another model of the association of fatigue, pain and depression is CFS. Recently, a review showed that chronic inflammation could explain, in part, the sickness behavior [57]. In this pathology, with increased sensitivity to pain and with sickness behavior, inflammatory cytokines could have an effect on nociception. Such cytokines appeared to be critical mediators of hyperalgesia in a lipopolysaccharide-induced animal model $[82,83]$. In CFS, the levels of neopterin, a marker of cellular immune system activation, IL-1 and TNF were correlated with fatigue and depression [84]. Brain inflammation could have a role in CFS, in part by activating microglia or astrocytes.
Indeed, the density of $11 \mathrm{C}$-(R)-(2-chlorophenyl)-N-methyl$\mathrm{N}$-(1-methylpropyl)-3-isoquinoline-carboxamide, a marker of neuroinflammation, was increased in some areas of the brain, in particular the cingulate cortex, in patients with CFS compared with healthy patients. Its increased level in the thalamus was correlated but not significantly with pain score and fatigue sensation $(P=0.0683)$ [85].

We have emerging evidence of the role of microbiota in the pathogenesis of autoimmune disease, particularly in rheumatologic disease [86]. Recently, Galland [87] proposed a schema in which the gut microbiome could affect CFS or fibromyalgia: the bacterial components could excessively stimulate the innate immune system and induce systemic and CNS inflammation by producing neurotoxic metabolites or could directly stimulate afferent neurons of the nervous system to send signals to the brain via the vagus nerve. Then, the gut microbiome could affect the HPA axis and be responsible for fatigue and pain in these diseases.

Moreover, it is interesting to highlight brain areas involved in these various domains. With the Kana Pickout Test, in the model of induced-fatigue previously described, the dorsolateral prefrontal cortex and cingulate cortex were activated as seen on functional MRI $[64,65]$. In adults, noxious stimulation increased MRI activity in primary somatosensory cortices, the anterior cingulate cortex, bilateral thalamus, and divisions of the insular cortices [88]. There is a common area for neural activity in experiences of physical pain, induced fatigue, depressive mood and bacterial endotoxin-induced inflammation (with increased IL-6 level): the anterior insula, believed to play a role in consciousness and in emotion or regulation of the body's homeostasis, and anterior cingulate cortex, known to be involved in autonomic and cognitive functions [64-66]. A common CNS pathway would be a link between fatigue and pain, and among the different mechanisms of fatigue, the action of inflammation on the CNS could be one of these pathways.

\section{Conclusion}

Fatigue and pain are two symptoms frequently present in acute or chronic high-grade inflammatory diseases such as infection, rheumatoid diseases or cancers but also low-grade inflammatory diseases such as CFS. These symptoms are often associated with depression. In all these diseases, several markers of inflammation have been highlighted, and among various and complex mechanisms of action, inflammation could be one of the common links between fatigue and pain among various and complex mechanisms. In rheumatic diseases, decreasing inflammation may improve fatigue and pain. Peripheral inflammation localized at organs is associated with central neurological phenomena. The inflammatory 
cytokines and cells interact with the CNS: peripheral inflammation can provoke fatigue and pain, and an altered neuroendocrine system could modulate inflammation. Because of multiple aspects of fatigue among patients and multiple mechanisms of action that could be involved, a future perspective would be to identify some phenotypes of fatigue to better target this treatment.

Note: This article is part of the series 'At the interface between immunology and neurology in rheumatic diseases', edited by Rainer Straub. Other articles in this series can be found at http://arthritis-research.com/series/neurology.

\section{Abbreviations}

BASDAl: Bath Ankylosing Spondylitis Disease Activity Index; CFS: Chronic fatigue syndrome; CNS: Central nervous system; COMT: Catechol-O-methyltransferase; CRP: C-reactive protein; DAS28: Disease Activity Score in 28 joints; DMARD: Disease-modifying antirheumatic drug; HPA: Hypothalamic-pituitaryadrenal; IFN: Interferon; IL: interleukin; IL-1RA: IL-1 receptor antagonist; MAF: Multidimensional Assessment of Fatigue; MRI: Magnetic resonance imaging; NFkB: Nuclear factor-kappa B; RA: Rheumatoid arthritis; SF: Short form; SpA: Spondyloarthritis; TNF: Tumor necrosis factor; VAS: Visual analog scale.

\section{Competing interests}

The authors declare that they have no competing interests.

\section{Published online: 05 October 2015}

\section{References}

1. Stebbings S, Treharne GJ. Fatigue in rheumatic disease: an overview. Int J Clin Rheumatol. 2010;5:487-502.

2. Nikolaus S, Bode C, Taal E, van de Laar MA. Fatigue and factors related to fatigue in rheumatoid arthritis: a systematic review. Arthritis Care Res (Hoboken). 2013;65:1128-46.

3. Bergman MJ, Shahouri SH, Shaver TS, Anderson JD, Weidensaul DN, Busch $R E$, et al. Is fatigue an inflammatory variable in rheumatoid arthritis (RA)? Analyses of fatigue in RA, osteoarthritis, and fibromyalgia. J Rheumatol. 2009:36:2788-94.

4. Pollard LC, Choy EH, Gonzalez J, Khoshaba B, Scott DL. Fatique in rheumatoid arthritis reflects pain, not disease activity. Rheumatology (Oxford). 2006:45:885-9.

5. van Dartel SA, Repping-Wuts JW, van Hoogmoed D, Bleijenberg G, van Riel PL, Fransen J. Association between fatigue and pain in rheumatoid arthritis: does pain precede fatigue or does fatigue precede pain? Arthritis Care Res (Hoboken). 2013;65:862-9.

6. Kirwan J, Heiberg T, Hewlett S, Hughes R, Kvien T, Ahlmen M, et al. Outcomes from the Patient Perspective Workshop at OMERACT 6. J Rheumatol. 2003;30:868-72.

7. Singh H, Arya S, Talapatra P, Lather K, Mathur R, Singhania A, et al. Assessment of fatigue in rheumatoid arthritis (by Functional Assessment of Chronic Illness Therapy-Fatigue score) and its relation to disease activity and anemia. J Clin Rheumatol. 2014;20:87-90.

8. Chauffier K, Paternotte S, Burki V, Durnez A, Elhai M, Koumakis E, et al. Fatigue in spondyloarthritis: a marker of disease activity. A cross-sectional study of 266 patients. Clin Exp Rheumatol. 2013:31:864-70.

9. Basu N, McClean A, Harper L, Amft EN, Dhaun N, Luqmani RA, et al. Explaining fatigue in ANCA-associated vasculitis. Rheumatology (Oxford). 2013;52:1680-5.

10. Lee YC, Frits ML, lannaccone CK, Weinblatt ME, Shadick NA, Williams DA, et al. Subgrouping of patients with rheumatoid arthritis based on pain, fatigue, inflammation, and psychosocial factors. Arthritis Rheumatol. 2014:66:2006-14.

11. Wolfe F, Hawley DJ, Wilson $K$. The prevalence and meaning of fatigue in rheumatic disease. J Rheumatol. 1996;23:1407-17.
12. Husted JA, Tom BD, Schentag CT, Farewell VT, Gladman DD. Occurrence and correlates of fatigue in psoriatic arthritis. Ann Rheum Dis. 2009;68:1553-8.

13. Hewlett S, Chalder T, Choy E, Cramp F, Davis B, Dures E, et al. Fatigue in rheumatoid arthritis: time for a conceptual model. Rheumatology (Oxford). 2011;50:1004-6.

14. Norheim KB, Jonsson G, Omdal R. Biological mechanisms of chronic fatigue. Rheumatology (Oxford). 2011:50:1009-18.

15. Rohleder N, Aringer M, Boentert M. Role of interleukin-6 in stress, sleep, and fatigue. Ann N Y Acad Sci. 2012;1261:88-96.

16. Nicassio PM, Moxham EG, Schuman CE, Gevirtz RN. The contribution of pain, reported sleep quality, and depressive symptoms to fatigue in fibromyalgia. Pain. 2002;100:271-9.

17. Neuberger GB, Press AN, Lindsley HB, Hinton R, Cagle PE, Carlson $K$, et al. Effects of exercise on fatigue, aerobic fitness, and disease activity measures in persons with rheumatoid arthritis. Res Nurs Health. 1997;20:195-204.

18. Rongen-van Dartel SA, Repping-Wuts $\mathrm{H}$, van Hoogmoed D, Knoop H, Bleijenberg G, van Riel PL, et al. Relationship between objectively assessed physical activity and fatigue in patients with rheumatoid arthritis: inverse correlation of activity and fatigue. Arthritis Care Res (Hoboken). 2014;66:852-60.

19. Stebbings SM, Treharne GJ, Jenks $K$, Highton J. Fatigue in patients with spondyloarthritis associates with disease activity, quality of life and inflammatory bowel symptoms. Clin Rheumatol. 2013:33:1467-74.

20. Hammoudeh M, Zack DJ, Li W, Stewart VM, Koenig AS. Associations between inflammation, nocturnal back pain and fatigue in ankylosing spondylitis and improvements with etanercept therapy. J Int Med Res. 2013;41:1150-9.

21. Goedendorp MM, Tack CJ, Steggink E, Bloot L, Bazelmans E, Knoop H. Chronic fatigue in type 1 diabetes: highly prevalent but not explained by hyperglycemia or glucose variability. Diabetes Care. 2014;37:73-80.

22. Willems LM, Kwakkenbos L, Leite CC, Thombs BD, van den Hoogen FH, Maia AC, et al. Frequency and impact of disease symptoms experienced by patients with systemic sclerosis from five European countries. Clin Exp Rheumatol. 2014;32:S-88-93.

23. Pellino G, Sciaudone G, Caserta V, Candilio G, De Fatico GS, Gagliardi S, et al. Fatigue in inflammatory bowel diseases: relationship with age and disease activity. Int J Surg. 2014;12:S60-3.

24. Meyers CA, Albitar M, Estey E. Cognitive impairment, fatigue, and cytokine levels in patients with acute myelogenous leukemia or myelodysplastic syndrome. Cancer. 2005;104:788-93.

25. Reyes-Gibby CC, Wang J, Spitz M, Wu X, Yennurajalingam S, Shete S. Genetic variations in interleukin-8 and interleukin-10 are associated with pain, depressed mood, and fatigue in lung cancer patients. J Pain Symptom Manage. 2013;46:161-72.

26. Miaskowski C, Cooper BA, Dhruva A, Dunn LB, Langford DJ, Cataldo JK, et al. Evidence of associations between cytokine genes and subjective reports of sleep disturbance in oncology patients and their family caregivers. PLOS One. 2012;7:e40560.

27. Irwin MR. Inflammation at the intersection of behavior and somatic symptoms. Psychiatr Clin North Am. 2013;34:605-20.

28. Bower JE. Cancer-related fatigue: links with inflammation in cancer patients and survivors. Brain Behav Immun. 2007;21:863-71.

29. Bower JE. Cancer-related fatigue - mechanisms, risk factors, and treatments Nat Rev Clin Oncol. 2014;11:597-609.

30. Illi J, Miaskowski C, Cooper B, Levine JD, Dunn L, West C, et al. Association between pro- and anti-inflammatory cytokine genes and a symptom cluster of pain, fatigue, sleep disturbance, and depression. Cytokine. 2012;58:437-47.

31. Bates DW, Schmitt W, Buchwald D, Ware NC, Lee J, Thoyer E, et al. Prevalence of fatigue and chronic fatigue syndrome in a primary care practice. Arch Intern Med. 1993:153:2759-65.

32. Bowman SJ, Hamburger J, Richards A, Barry RJ, Rauz S. Patient-reported outcomes in primary Sjogren's syndrome: comparison of the long and short versions of the Profile of Fatigue and Discomfort - Sicca Symptoms Inventory. Rheumatology (Oxford). 2009;48:140-3.

33. Jump RL, Robinson ME, Armstrong AE, Barnes EV, Kilbourn KM, Richards HB. Fatigue in systemic lupus erythematosus: contributions of disease activity, pain, depression, and perceived social support. J Rheumatol. 2005;32:1699-705.

34. Grayson PC, Amudala NA, McAlear CA, Leduc RL, Shereff D, Richesson R, et al. Illness perceptions and fatigue in systemic vasculitis. Arthritis Care Res (Hoboken). 2013;65:1835-43.

35. Gossec L, Berenbaum F, Chauvin P, Lamiraud K, Russo-Marie F, Joubert JM, et al. Reporting of patient-perceived impact of rheumatoid arthritis and axial spondyloarthritis over 10 years: a systematic literature review. Rheumatology (Oxford). 2014;53:1274-81. 
36. Repping-Wuts $H$, Repping $T$, van Riel $P$, van Achterberg T. Fatigue communication at the out-patient clinic of Rheumatology. Patient Educ Couns. 2009;76:57-62.

37. Repping-Wuts H, Fransen J, van Achterberg T, Bleijenberg G, van Riel P. Persistent severe fatigue in patients with rheumatoid arthritis. J Clin Nurs. 2007:16:377-83.

38. van Hoogmoed D, Fransen J, Bleijenberg G, van Riel P. Physical and psychosocial correlates of severe fatigue in rheumatoid arthritis. Rheumatology (Oxford). 2010;49:1294-302.

39. Haywood KL, Packham JC, Jordan KP. Assessing fatigue in ankylosing spondylitis: the importance of frequency and severity. Rheumatology (Oxford). 2013;53:552-6.

40. Hewlett S, Hehir M, Kirwan JR. Measuring fatigue in rheumatoid arthritis: a systematic review of scales in use. Arthritis Rheum. 2007;57:429-39.

41. Smets EM, Garssen B, Bonke B, De Haes JC. The Multidimensional Fatigue Inventory (MFI) psychometric qualities of an instrument to assess fatigue. J Psychosom Res. 1995;39:315-25.

42. Mendoza TR, Wang XS, Cleeland CS, Morrissey M, Johnson BA, Wendt JK, et al. The rapid assessment of fatigue severity in cancer patients: use of the Brief Fatigue Inventory. Cancer. 1999:85:1186-96.

43. Wolfe F. Fatigue assessments in rheumatoid arthritis: comparative performance of visual analog scales and longer fatigue questionnaires in 7760 patients. J Rheumatol. 2004;31:1896-902.

44. Conigliaro P, Triggianese P, Ippolito F, Lucchetti R, Chimenti MS, Perricone R. Insights on the role of physical activity in patients with rheumatoid arthritis. Drug Dev Res. 2014;75:S54-6.

45. Scott DL. Leflunomide improves quality of life in rheumatoid arthritis. Scand J Rheumatol Suppl. 1999;112:23-9.

46. Chauffier K, Salliot C, Berenbaum F, Sellam J. Effect of biotherapies on fatigue in rheumatoid arthritis: a systematic review of the literature and meta-analysis. Rheumatology (Oxford). 2012;51:60-8.

47. Strand V, Burmester GR, Zerbini CA, Mebus CA, Zwillich SH, Gruben D, et al. Tofacitinib with methotrexate in third-line treatment of patients with active rheumatoid arthritis: patient-reported outcomes from a Phase 3 trial. Arthritis Care Res (Hoboken). 2015;67:475-83.

48. Strand V, Kosinski M, Gnanasakthy A, Mallya U, Mpofu S. Secukinumab treatment in rheumatoid arthritis is associated with incremental benefit in the clinical outcomes and HRQOL improvements that exceed minimally important thresholds. Health Qual Life Outcomes. 2014;12:31.

49. Wells G, Li T, Maxwell L, Maclean R, Tugwell P. Responsiveness of patient reported outcomes including fatigue, sleep quality, activity limitation, and quality of life following treatment with abatacept for rheumatoid arthritis. Ann Rheum Dis. 2008;67:260-5.

50. Minnock P, Kirwan J, Bresnihan B. Fatigue is a reliable, sensitive and unique outcome measure in rheumatoid arthritis. Rheumatology (Oxford). 2009;48:1533-6.

51. Durcan L, Wilson F, Cunnane G. The effect of exercise on sleep and fatigue in rheumatoid arthritis: a randomized controlled study. J Rheumatol. 2014;41:1966-73.

52. Hewlett S, Ambler N, Almeida C, Cliss A, Hammond A, Kitchen K, et al. Self-management of fatigue in rheumatoid arthritis: a randomised controlled trial of group cognitive-behavioural therapy. Ann Rheum Dis. 2011;70:1060-7.

53. Garip Y, Eser F, Aktekin LA, Bodur H. Fatigue in rheumatoid arthritis: association with severity of pain, disease activity and functional status. Acta Reumatol Port. 2011;36:364-9.

54. Huyser BA, Parker JC, Thoreson R, Smarr KL, Johnson JC, Hoffman R. Predictors of subjective fatigue among individuals with rheumatoid arthritis. Arthritis Rheum. 1998;41:2230-7.

55. Norden DM, Bicer S, Clark Y, Jing R, Henry CJ, Wold LE, et al. Tumor growth increases neuroinflammation, fatigue and depressive-like behavior prior to alterations in muscle function. Brain Behav Immun. 2014;43:76-85.

56. Bluthe RM, Beaudu C, Kelley KW, Dantzer R. Differential effects of IL-1 ra on sickness behavior and weight loss induced by IL-1 in rats. Brain Res. 1995;677:171-6.

57. Arnett SV, Clark IA. Inflammatory fatigue and sickness behaviour - lessons for the diagnosis and management of chronic fatigue syndrome. J Affect Disord. 2012;141:130-42.

58. Neu D, Mairesse O, Montana X, Gilson M, Corazza F, Lefevre N, et al. Dimensions of pure chronic fatigue: psychophysical, cognitive and biological correlates in the chronic fatigue syndrome. Eur J Appl Physiol. 2014;114:1841-51.
59. Heinzelmann M, Lee H, Rak H, Livingston W, Barr T, Baxter T, et al. Sleep restoration is associated with reduced plasma C-reactive protein and depression symptoms in military personnel with sleep disturbance after deployment. Sleep Med. 2014;15:1565-70.

60. Masson C. Rheumatoid anemia. Joint Bone Spine. 2011;78:131-7.

61. Chrousos GP. The hypothalamic-pituitary-adrenal axis and immune-mediated inflammation. N Engl J Med. 2010;332:1351-62.

62. Sprangers MA, Thong MS, Bartels M, Barsevick A, Ordonana J, Shi Q, et al. Biological pathways, candidate genes, and molecular markers associated with quality-of-life domains: an update. Qual Life Res. 2014;23:1997-2013.

63. Dantzer R, Heijnen CJ, Kavelaars A, Laye S, Capuron L. The neuroimmune basis of fatigue. Trends Neurosci. 2014;37:39-46.

64. Mizuno K, Tajima K, Watanabe Y, Kuratsune H. Fatigue correlates with the decrease in parasympathetic sinus modulation induced by a cognitive challenge. Behav Brain Funct. 2014;10:25.

65. Tachibana A, Noah JA, Bronner S, Ono Y, Hirano Y, Niwa M, et al. Activation of dorsolateral prefrontal cortex in a dual neuropsychological screening test: an fMRI approach. Behav Brain Funct. 2012;8:26.

66. Slavich GM, Irwin MR. From stress to inflammation and major depressive disorder: a social signal transduction theory of depression. Psychol Bull. 2014;140:774-815.

67. Thomson CA, McColl A, Cavanagh J, Graham GJ. Peripheral inflammation is associated with remote global gene expression changes in the brain. J Neuroinflammation. 2014;11:73.

68. Anisman H. Inflaming depression. J Psychiatry Neurosci. 2011;36:291-5.

69. Tweedie D, Sambamurti K, Greig NH. TNF-alpha inhibition as a treatment strategy for neurodegenerative disorders: new drug candidates and targets. Curr Alzheimer Res. 2007;4:378-85.

70. Schaible HG. Nociceptive neurons detect cytokines in arthritis. Arthritis Res Ther. 2014:16:470.

71. Richter F, Natura G, Loser S, Schmidt K, Viisanen H, Schaible HG. Tumor necrosis factor causes persistent sensitization of joint nociceptors to mechanical stimuli in rats. Arthritis Rheum. 2010;62:3806-14.

72. Walsh DA, McWilliams DF. Mechanisms, impact and management of pain in rheumatoid arthritis. Nat Rev Rheumatol. 2014;10:581-92.

73. Dowlati Y, Herrmann N, Swardfager W, Liu H, Sham L, Reim EK, et al. A metaanalysis of cytokines in major depression. Biol Psychiatry. 2010;67:446-57.

74. Zalli A, Jovanova O, Hoogendijk WJ, Tiemeier H, Carvalho LA. Low-grade inflammation predicts persistence of depressive symptoms. Psychopharmacology (Berl). 2015. [Epub ahead of print].

75. lyengar RL, Gandhi S, Aneja A, Thorpe K, Razzouk L, Greenberg J, et al. NSAIDs are associated with lower depression scores in patients with osteoarthritis. Am J Med. 2013;126:1017. e11-8.

76. Basterzi AD, Aydemir C, Kisa C, Aksaray S, Tuzer V, Yazici K, et al. IL-6 levels decrease with SSRI treatment in patients with major depression. Hum Psychopharmacol. 2005;20:473-6.

77. Lasselin J, Capuron L. Chronic low-grade inflammation in metabolic disorders: relevance for behavioral symptoms. Neuroimmunomodulation. 2014;21:95-101.

78. Lampa J, Westman M, Kadetoff D, Agreus AN, Le Maitre E, Gillis-Haegerstrand C, et al. Peripheral inflammatory disease associated with centrally activated IL-1 system in humans and mice. Proc Natl Acad Sci U S A. 2012;109:12728-33.

79. Genovese MC, McKay JD, Nasonov EL, Mysler EF, da Silva NA, Alecock E, et al. Interleukin- 6 receptor inhibition with tocilizumab reduces disease activity in rheumatoid arthritis with inadequate response to diseasemodifying antirheumatic drugs: the tocilizumab in combination with traditional disease-modifying antirheumatic drug therapy study. Arthritis Rheum. 2008:58:2968-80.

80. Laird BJ, McMillan DC, Fayers P, Fearon K, Kaasa S, Fallon MT, et al. The systemic inflammatory response and its relationship to pain and other symptoms in advanced cancer. Oncologist. 2013;18:1050-5.

81. Schrepf A, Clevenger L, Christensen D, DeGeest K, Bender D, Ahmed A, et al Cortisol and inflammatory processes in ovarian cancer patients following primary treatment: relationships with depression, fatigue, and disability. Brain Behav Immun. 2013;30:S126-34.

82. Watkins LR, Wiertelak EP, Goehler LE, Smith KP, Martin D, Maier SF. Characterization of cytokine-induced hyperalgesia. Brain Res. 1994;654:15-26.

83. Maier SF, Wiertelak EP, Martin D, Watkins LR. Interleukin-1 mediates the behavioral hyperalgesia produced by lithium chloride and endotoxin. Brain Res. 1993;623:321-4. 
84. Maes M, Twisk FN, Kubera M, Ringel K. Evidence for inflammation and activation of cell-mediated immunity in myalgic encephalomyelitis/chronic fatigue syndrome (ME/CFS): increased interleukin-1, tumor necrosis factoralpha, PMN-elastase, lysozyme and neopterin. J Affect Disord. 2012;136:933-9.

85. Nakatomi Y, Mizuno K, Ishii A, Wada Y, Tanaka M, Tazawa S, et al.

Neuroinflammation in patients with chronic fatigue syndrome/myalgic encephalomyelitis: an 11C-(R)-PK1 1195 PET Study. J Nucl Med. 2014;55:945-50.

86. McLean MH, Dieguez Jr D, Miller LM, Young HA. Does the microbiota play a role in the pathogenesis of autoimmune diseases? Gut. 2015;64:332-41.

87. Galland L. The gut microbiome and the brain. J Med Food. 2014;17:1261-72.

88. Goksan S, Hartley C, Emery F, Cockrill N, Poorun R, Moultrie F, et al. fMRI reveals neural activity overlap between adult and infant pain. Elife. 2015;4. doi:10.7554/eLife.06356. 